\title{
Quantum Zeno effect appears in stages
}

\author{
Kyrylo Snizhko $\odot,{ }^{1}$ Parveen Kumar $\odot,{ }^{1}$ and Alessandro Romito $\odot^{2}$ \\ ${ }^{1}$ Department of Condensed Matter Physics, Weizmann Institute of Science, Rehovot 76100, Israel \\ ${ }^{2}$ Department of Physics, Lancaster University, Lancaster LA1 4YB, England, United Kingdom
}

(Received 25 March 2020; accepted 13 August 2020; published 29 September 2020)

\begin{abstract}
In the quantum Zeno effect, quantum measurements can block the coherent oscillation of a two level system by freezing its state to one of the measurement eigenstates. The effect is conventionally controlled by the measurement frequency. Here we study the development of the Zeno regime as a function of the measurement strength for a continuous partial measurement. We show that the onset of the Zeno regime is marked by a cascade of transitions in the system dynamics as the measurement strength is increased. Some of these transitions are only apparent in the collective behavior of individual quantum trajectories and are invisible to the average dynamics. They include the appearance of a region of dynamically inaccessible states and of singularities in the steady-state probability distribution of states. These predicted dynamical features, which can be readily observed in current experiments, show the coexistence of fundamentally unpredictable quantum jumps with those continuously monitored and reverted in recent experiments.
\end{abstract}

DOI: 10.1103/PhysRevResearch.2.033512

\section{INTRODUCTION}

The evolution of a quantum system under measurement is inherently stochastic due to the intrinsic quantum fluctuations of the detector [1]. If these fluctuations can be accurately monitored, measurements can be used to track the stochastic evolution of the system state, i.e., individual quantum trajectories. From a theoretical tool to investigate open quantum systems [2], quantum trajectories have become an observable reality in experiments in optical [3,4] and solid-state [5-7] systems. Tracking quantum trajectories has been exploited as a tool to engineering quantum states via continuous feedback control [8-10] and entanglement distillation [11,12]. It has been used to observe fundamental properties of quantum measurements [13-17] and, recently, to predict topological transitions in measurement-induced geometric phases [18-21] and many-body entanglement phase transitions in random unitary circuits, invisible to the average dynamics [22-25]. Monitoring quantum trajectories has also made possible anticipating and correcting quantum jumps in superconducting qubits [26].

The above-mentioned transitions stem from the basic physics of the quantum Zeno effect [27,28]. In this regime, as a result of repeated measurements, the system state is mostly frozen next to one of the measurement eigenstates, yet rarely performs quantum jumps between them. The crossover between coherent oscillations and the Zeno regime is controlled by the frequency of the measurement and has been

Published by the American Physical Society under the terms of the Creative Commons Attribution 4.0 International license. Further distribution of this work must maintain attribution to the author(s) and the published article's title, journal citation, and DOI. extensively explored both theoretically [29-34] and experimentally [35-39]. Beyond projective measurements, the onset of the Zeno regime is richer [40,41], and quantum jumps appear as part of continuous stochastic dynamics. For example, in a system monitored via continuous partial measurements, quantum jumps can be anticipated, continuously monitored, and reverted [26], a task which is fundamentally impossible with projective measurements. Moreover, the onset of the Zeno regime with nonprojective measurements is more convoluted and has been characterized by different measurement strengths and phenomenology based on the dynamics of the detector signal [42], average [43-47], or postselected [48,49] state evolution.

Here we study the transition between the regimes of coherent oscillations and Zeno-like dynamics in a qubit subject to continuous partial measurements [see Fig. 1(a)], a model directly describing some recent experiments [26]. By investigating the full stochastic dynamics of quantum trajectories, we show that the quantum Zeno regime is established via a cascade of transitions in the system dynamics, some being invisible to the average dynamics. Furthermore, we find that, in the Zeno regime, catchable continuous jumps between states $|1\rangle$ and $|0\rangle$ necessarily have a discontinuous counterpart, jumps between $|0\rangle$ and $|1\rangle$, which are inherently unpredictable in individual realizations. Our results provide a unified picture of the onset of the Zeno regime arising from continuous partial measurements and demonstrate that investigating individual quantum trajectories can uncover drastically new physics even in simple and well-studied systems. Our findings may be relevant for quantum error correction protocols employing continuous partial measurements [50,51].

\section{MODEL AND POSTSELECTED DYNAMICS}

We consider a qubit performing coherent quantum oscillations between states $|0\rangle$ and $|1\rangle$ due to the Hamiltonian 


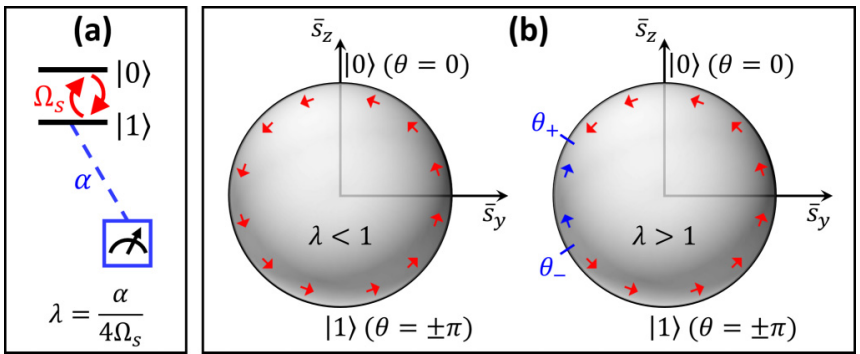

FIG. 1. (a) The system. A Hamiltonian induces oscillations between levels $|0\rangle$ and $|1\rangle$ of a qubit, which is continuously measured by a detector weakly coupled to one of the levels. (b) Dynamical flow (red and blue arrows) of $\theta(t)$ from Eq. (3) under "no-click" postselected dynamics. For sufficiently weak measurements, $\lambda<1$ (left), the dynamics is oscillatory; for $\lambda>1$ (right), stable and unstable fixed points $\left(\theta_{+}\right.$and $\theta_{-}$, respectively) emerge. The states in the interval $\theta \in\left(-\pi, \theta_{+}\right)$are inaccessible to the system under both the no-click and the full stochastic dynamics.

$H_{s}=\Omega_{s} \sigma_{x}$, where $\Omega_{s}>0$; at the same time the qubit is monitored by a sequence of measurements at intervals $d t \ll 1 / \Omega_{s}$ [see Fig. 1(a)]. Each measurement is characterized by two possible readouts $r=0$ ("no-click") and 1 ("click"). The corresponding measurement back-action $|\psi\rangle \rightarrow M^{(r)}|\psi\rangle$ is given by the operators

$$
M^{(0)}=|0\rangle\langle 0|+\sqrt{1-p}| 1\rangle\left\langle 1\left|, \quad M^{(1)}=\sqrt{p}\right| 1\right\rangle\langle 1|,
$$

where $p \in[0,1]$ controls the measurement strength. For $p=$ 1 , each measurement is projective and this induces the conventional quantum Zeno effect with the system being frozen in one of the measurement eigenstates, $|0\rangle$ or $|1\rangle$. In the opposite limit, $p=0$, essentially no measurement takes place, and the system performs Rabi oscillations under $H_{s}$. We investigate the intermediate case of $p=\alpha d t$ with $d t \rightarrow 0$, and $\alpha \geqslant 0$ controlling the effective measurement strength over a finite time interval. A physical model of this measurement process is realized by coupling the system to a two-level system detector that, in turn, is subject to projective measurements (see Appendix A for details).

In each infinitesimal step the measurement and the system evolution add up to give the combined evolution

$$
|\psi(t+d t)\rangle=M^{(r)} U|\psi(t)\rangle,
$$

where $U=e^{-i H_{s} d t} \approx 1-i H_{s} d t$ is the Hamiltonian unitary evolution over an infinitesimal time interval $d t$. When the system is initialized in $|0\rangle$ or $|1\rangle$, its evolution is constrained to the $y-z$ section of the Bloch sphere and the state has the form $|\psi(t)\rangle=|\psi[\theta(t)]\rangle=\cos \frac{\theta(t)}{2}|0\rangle+i \sin \frac{\theta(t)}{2}|1\rangle$. Equation (2) translates onto

$$
\theta(t+d t)=\left\{\begin{array}{ll}
\theta(t)-\Omega[\theta(t)] d t & \text { if } r=0 \\
\pi & \text { if } r=1
\end{array},\right.
$$

where $\Omega(\theta)=2 \Omega_{s}[1+\lambda \sin \theta]$ and $\lambda=\frac{\alpha}{4 \Omega_{s}}$ sets the strength of the measurement relative to the Hamiltonian. A measurement yielding readout $r=1$ immediately projects the system onto state $|1\rangle$, while a no-click $r=0$ readout implies an infinitesimal evolution of the state with angular velocity $\Omega(\theta)$.
The probabilities of the two possible readouts are given by

$$
p_{r=1} \equiv p_{1}=\alpha d t \sin ^{2} \frac{\theta(t)}{2}, \quad p_{r=0} \equiv p_{0}=1-p_{1}
$$

and depend on the qubit state, i.e., on $\theta(t)$.

For understanding the full stochastic dynamics, it is instructive to review its continuous no-click part, previously analyzed in Ref. [48]. In this case the state evolution is governed by the the differential equation $\dot{\theta}=-\Omega(\theta)$. The corresponding flow of the variable $\theta$ is shown in Fig. 1(b). Since $\Omega_{s}>0$ and $\lambda>0$, for any $\theta \in(0, \pi)$, we have $\Omega(\theta)>$ 0 , and the system evolves continuously towards $\theta=0$. Notably, this is the only way for the system state to evolve from $|1\rangle$ to $|0\rangle$ and it corresponds to the quantum jumps that have been continuously monitored in Ref. [26]. The transition from $|0\rangle$ to $|1\rangle$, instead, takes place via the region $\theta \in(-\pi, 0)$ and has richer dynamics controlled by the measurement strength. For sufficiently weak measurements, $0 \leqslant \lambda<1$, one has $\Omega(\theta)>0$ for any $\theta$, and the system monotonously evolves towards $\theta=-\pi$; however, for $\lambda>1$ there appear two fixed points, $\Omega\left(\theta_{ \pm}\right)=0$, at

$$
\theta_{ \pm}=2 \arctan \left(-\lambda \pm \sqrt{\lambda^{2}-1}\right)
$$

where $\theta_{+}$is a stable point, while $\theta_{-}$is an unstable one, as shown in Fig. 1(b). Under the $r=0$ postselected dynamics for $\lambda>1$, the system will eventually flow to $\theta=\theta_{+}$[48] (where it remains until the occurrence of a click, which collapses the system to $|1\rangle)$.

\section{STOCHASTIC EVOLUTION AND DYNAMICAL TRANSITIONS}

Beyond the postselected $r=0$ quantum trajectory, the stochastic dynamics of the system is described by the probability density $P_{t}(\theta)$ of being in the state $|\psi(\theta)\rangle$ at time $t$. Using Eqs. (3) and (4), one derives the master equation for $P_{t}(\theta)$ :

$$
\begin{aligned}
\frac{d P_{t}(\theta)}{d t}= & {\left[\partial_{\theta}\left[\Omega(\theta) P_{t}(\theta)\right]-4 \Omega_{s} \lambda \sin ^{2} \frac{\theta}{2} P_{t}(\theta)\right.} \\
& \left.+4 \Omega_{s} \lambda \delta(\theta-\pi) \int_{0}^{2 \pi} d \tilde{\theta} \sin ^{2} \frac{\tilde{\theta}}{2} P_{t}(\tilde{\theta})\right] .
\end{aligned}
$$

Here, the first term on the right-hand side describes the no-click evolution, the second term describes the reduction of $P_{t}(\theta)$ due to clicks that happen with probability $p_{1}=$ $4 \Omega_{s} \lambda \sin ^{2} \frac{\theta}{2} d t$ [see Eq. (4)], while the last term accounts for the clicks bringing the states from any $\theta$ to $\theta=\pi$.

Two experimentally accessible quantities directly related to $P_{t}(\theta)$ capture the main physics: the steady-state distribution $P_{\infty}(\theta) \equiv \lim _{t \rightarrow \infty} P_{t}(\theta)$ and the average "polarization" of the qubit, $\overline{\boldsymbol{s}}(t) \equiv\left(\bar{s}_{y}(t), \bar{s}_{z}(t)\right)$, where $\bar{s}_{i}(t) \equiv\left\langle\sigma_{i}(t)\right\rangle=$ $\int_{-\pi}^{\pi}\left\langle\psi(\theta)\left|\sigma_{i}\right| \psi(\theta)\right\rangle P_{t}(\theta) d \theta, \quad i=y, z$. Both quantities are plotted in Fig. 2. They showcase three qualitative transitions in the dynamics as function of the measurement strength.

We can readily present the key physics of these transitions before entering all the features in due details. For sufficiently small $\lambda$, the qubit can be found in any state with finite probability density $P_{\infty}(\theta) \neq 0$. In particular, it is possible to evolve from $|0\rangle$ to $|1\rangle$ via trajectories involving a detector click as 


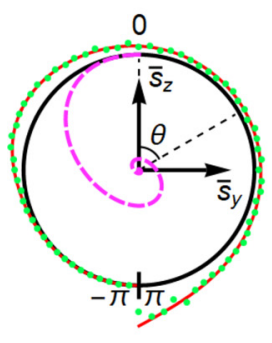

$\lambda=0.7$

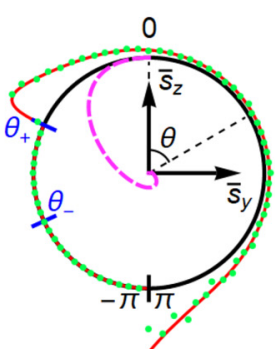

$\lambda=1.1$

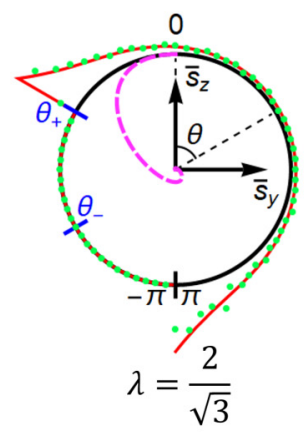

- $P_{t=\infty}(\theta)$ (Monte Carlo numerics)

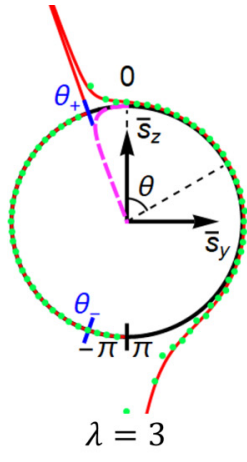

$--\overline{\boldsymbol{s}}(t)$

FIG. 2. The stochastic dynamics of the qubit state in the $y-z$ section of the Bloch sphere exhibits transitions at relative measurement strengths $\lambda=1,2 / \sqrt{3}$, and 2 . The long-time probability distribution $P_{t=\infty}(\theta)$ at different values of $\lambda$ is shown as the height above the unit circle for the analytic result (red solid line) and for the numerical simulations (green dots). The trajectories of the expectation values $\bar{s}_{y, z}(t)$, for the system initialized in $|0\rangle$ at $t=0$, are shown with the dashed magenta lines. Each numerical simulation involved 10000 stochastic trajectory realizations, tracing the evolution until $t=10$, with measurements yielding random outcomes happening at intervals $d t=0.01$, using $\Omega_{s}=1$; $\theta$ is binned in intervals of size $5^{\circ}$.

well as no clicks from the detector. Instead, the evolution from $|1\rangle$ to $|0\rangle$ happens only via no-click sequences, as noted above. The first and most drastic transition happens at $\lambda=1$, above which there opens a region of $\theta \in\left(-\pi ; \theta_{+}\right]$where $P_{\infty}(\theta)=0$. In fact, this region is inaccessible for the qubit at any time $t$; hence, for $\lambda>1$ all quantum trajectories from $|0\rangle$ to $|1\rangle$ must involve a detector click. Generically, the click may occur when the qubit has not reached $\theta_{+}$, which is typically the case. The second transition happens at $\lambda=2 / \sqrt{3}$, above which $P_{\infty}(\theta)$ diverges at $\theta=\theta_{+}$. This indicates that the system initialized in $|0\rangle$ typically reaches the vicinity of $\theta_{+}$, and spends a long time there, before the click and the corresponding jump to $\theta=\pi$ take place. So far, the population imbalance between $|0\rangle$ and $|1\rangle, \bar{s}_{z}(t)$, exhibits oscillations, which are reflected in the oscillations of the average state polarization, $\bar{s}(t)$. The third and final transition at $\lambda=2$ marks the end of the oscillations, so that, for $\lambda>2, \bar{s}_{z}(t)$ steadily decays in time, completing the final onset of Zeno-like dynamics. These transitions set the overall picture of the onset of the Zeno regime in the system, and constitute the main findings of our paper.

To analyze these transitions and their implications in some detail, consider first the nontrivial steady state, $P_{\infty}(\theta)$. From the condition $d P_{t \rightarrow \infty}(\theta) / d t=0$, Eq. (6) gives

$$
P_{\infty}(\theta)=\frac{\lambda \exp \left[\frac{2 \lambda}{\sqrt{1-\lambda^{2}}}\left(\arctan \frac{\lambda+\tan \frac{\theta}{2}}{\sqrt{1-\lambda^{2}}}-\frac{\pi}{2}\right)\right]}{(1+\lambda \sin \theta)^{2}\left[1-\exp \left(-\frac{2 \pi \lambda}{\sqrt{1-\lambda^{2}}}\right)\right]},
$$

for $\lambda<1$, while for $\lambda>1$ the expression reads

$$
P_{\infty}(\theta)= \begin{cases}\frac{\lambda}{(1+\lambda \sin \theta)^{2}}\left(\frac{\tan \frac{\theta}{2}+\lambda-\sqrt{\lambda^{2}-1}}{\tan \frac{\theta}{2}+\lambda+\sqrt{\lambda^{2}-1}}\right)^{\frac{\lambda}{\sqrt{\lambda^{2}-1}}}, & \theta \in\left(\theta_{+} ; \pi\right], \\ 0, & \theta \in\left(-\pi ; \theta_{+}\right] .\end{cases}
$$

In Fig. 2, the analytical results in Eqs. (7) and (8) are compared with Monte Carlo numerical simulations of individual quantum trajectories, showing excellent agreement. The first two transitions in the system dynamics highlighted above are evident from $P_{\infty}(\theta)$ (see Fig. 2). The opening of the forbidden region $\left(-\pi ; \theta_{+}\right]$appears discontinuously rather than opening smoothly, since $\theta_{+}=\theta_{-}=-\pi / 2$ at $\lambda=1$. It manifests itself in the nonanalytic behavior of $P_{\infty}(\theta)$ as a function of $\lambda$ at $\lambda=1$. For $\lambda>1$, the second transition shows up at $\theta \approx \theta_{+}$, where

$$
P_{\infty}(\theta) \propto\left(\tan \frac{\theta}{2}-\tan \frac{\theta_{+}}{2}\right)^{\frac{\lambda}{\sqrt{\lambda^{2}-1}}-2},
$$

which diverges at $\theta=\theta_{+}$for $\lambda>2 / \sqrt{3}$. The physics underpinning this transition is evinced by Eq. (6) at $\theta=\theta_{+}$. The first two terms in the right-hand side, $P_{t}\left(\theta_{+}\right) \partial_{\theta_{+}} \Omega\left(\theta_{+}\right)$ and $-4 \Omega_{s} \lambda \sin ^{2} \frac{\theta_{+}}{2} P_{t}\left(\theta_{+}\right)$, describe the rate of accumulation of probability for states at $\theta \approx \theta_{+}$due to no-click dynamics and the loss of such probability due to detector clicks, respectively. At $\lambda=2 / \sqrt{3}$ the two terms balance each other, and for $\lambda>2 / \sqrt{3}$ the former dominates. Note that the $\lambda=2 / \sqrt{3}$ transition goes unnoticed in both the average [state polarization $\bar{s}(t)]$ behavior and in the postselected $r=0$ dynamics.

While the steady-state properties of $P_{\infty}(\theta)$ showcase these transitions, their role in the onset of the Zeno regime is fully unveiled only in the full stochastic dynamics. To appreciate that, consider the probability $P^{(0)}(t)$ of obtaining a sequence of no clicks ( $r=0$ readouts) of duration $t$ [see Fig. 3 (inset)]. We obtain that at long times $P^{(0)}(t)$ decays exponentially as

$$
P^{(0)}(t) \propto e^{-2 \Omega_{s} \zeta(\lambda) t}\{A(\lambda)+B(\lambda) \cos [\varepsilon t+\varphi(\lambda)]\},
$$

where $B(\lambda)=0$ for $\lambda>1$, the frequency of the oscillatory term for $\lambda<1$ is $\varepsilon=2 \Omega_{s} \sqrt{1-\lambda^{2}}$, and the decay rate is $\zeta(\lambda)=\operatorname{Re}\left[\lambda-\sqrt{\lambda^{2}-1}\right]$ (see Appendix B for the derivation). The value $\lambda=1$ is special in two respects. For $\lambda<1$, the system state rotates between $|0\rangle$ and $|1\rangle$ under no-click dynamics. However, the probability of observing a click is different for different $\theta$-hence the oscillations of $P^{(0)}(t)$. With the appearance of the forbidden region, the evolution under no-click readout is frozen at $\theta=\theta_{+}$, hence the oscillations of $P^{(0)}(t)$ disappear. The less obvious effect is that at $\lambda=1$ the decay rate $\zeta(\lambda)$ is maximal (see Fig. 3). Therefore, the 


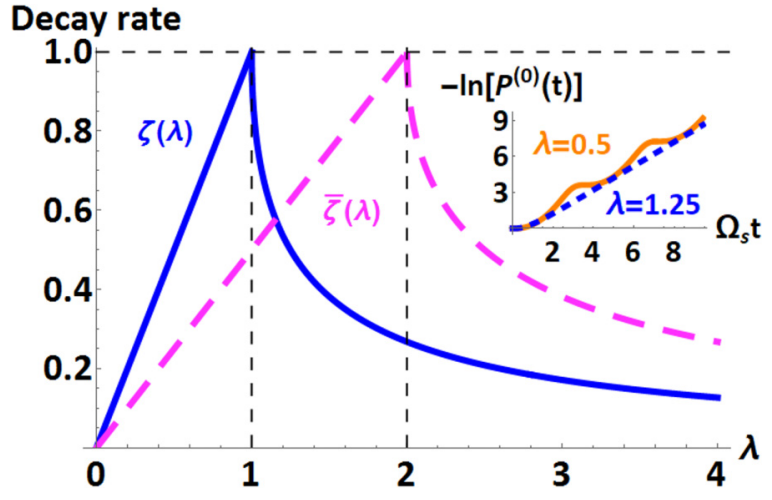

FIG. 3. The decay rates $\zeta(\lambda)$ (solid) and $\bar{\zeta}(\lambda)$ (dashed) characterizing, respectively, the probability to observe no clicks $P^{(0)}(t)$ and the survival probability $\left[1+\bar{s}_{z}(t)\right] / 2$. Note the respective decay rate maxima at $\lambda=1$ and 2. Inset: The time dependence of $P^{(0)}(t)$ for $\lambda=0.5$ (solid) and $\lambda=1.25$ (dashed). At long times $P^{(0)}(t)$ decays exponentially with (without) superimposed oscillations for $\lambda<1$ $(\lambda>1)$.

probability of observing a long sequence of no clicks increases with the measurement strength for $\lambda \geqslant 1$, while it decreases for $\lambda \leqslant 1$.

Consider now the probability $P^{(0)}(\theta)$ to reach a particular value of $\theta$ under no-click dynamics. $P^{(0)}(\theta)$ is obtained from $P^{(0)}(t)$ and the no-click evolution $\dot{\theta}(t)=-\Omega[\theta(t)]$ via $P^{(0)}\left(\theta_{0}\right) \equiv P^{(0)}\left(t_{0}\right)$ with $t_{0}$ satisfying $\theta\left(t_{0}\right)=\theta_{0}$. In proximity of $\theta=\theta_{+}$, one has (see Appendix B)

$$
P^{(0)}\left(\theta \approx \theta_{+}\right) \propto\left(\tan \frac{\theta}{2}-\tan \frac{\theta_{+}}{2}\right)^{\frac{\lambda}{\sqrt{\lambda^{2}-1}}-1} .
$$

Note that $P^{(0)}(\theta)$ vanishes at $\theta=\theta_{+}$for any finite $\lambda>1$. However, $d P^{(0)}(\theta) / d \theta$ vanishes at $\theta_{+}$for $\lambda<2 / \sqrt{3}$ and diverges for $\lambda>2 / \sqrt{3}$. Therefore, for $\lambda<2 / \sqrt{3}$, the system typically jumps to $\theta=\pi$ via a detector click before it reaches $\theta_{+}$. For $\lambda>2 / \sqrt{3}$, the system is likely to reach a close vicinity of $\theta_{+}$before a click happens.

To observe the last transition, $\lambda=2$, one needs to consider the average state polarization, $\bar{s}(t)=\left(\bar{s}_{y}(t), \bar{s}_{z}(t)\right)$, and in particular the population imbalance, $\bar{s}_{z}(t)$. When the system is initialized in state $|0\rangle$ at time $t=0$, using Eq. (6), one finds (see Appendix B)

$\bar{s}_{z}(t)=e^{-\Omega_{s} \lambda t}\left(\cosh \Omega_{s} t \sqrt{\lambda^{2}-4}+\lambda \frac{\sinh \Omega_{s} t \sqrt{\lambda^{2}-4}}{\sqrt{\lambda^{2}-4}}\right)$.

One sees that $\lambda=2$ marks a transition from oscillatory (at $\lambda<2$ ) to nonoscillatory (at $\lambda>2$ ) dynamics. The same transition is observed in $\bar{s}_{y}(t)$. Similarly to $P^{(0)}(t)$ in Eq. (10), $\bar{s}_{z}(t \rightarrow+\infty) \propto e^{-2 \Omega_{s} \bar{\zeta}(\lambda) t}$ with the decay rate $\bar{\zeta}(\lambda)=\operatorname{Re}\left[\lambda-\sqrt{\lambda^{2}-4}\right] / 2$. Therefore, the decay rate exhibits a maximum at $\lambda=2$ (see Fig. 3). Importantly, $\bar{s}_{z}(t)$ characterizes not only the population imbalance but also the survival probability $\left[1+\bar{s}_{z}(t)\right] / 2$, i.e., the probability to find the system in state $|0\rangle$ when performing a projective measurement at time $t$. The decay rate behavior implies that the long-time survival probability increases with increasing $\lambda$ when $\lambda \geqslant 2$, and decreases otherwise [52]. On this ground, one marks the transition at $\lambda=2$ as the final onset of the Zeno-like dynamics. Notably, the $\lambda=2$ transition is also reflected in a topological transition in the statistics of the detector clicks [42].

We would like to note that while we used different quantities to showcase each of the three dynamical transitions all three of them can be inferred by looking at a single quantity: the eigenmode spectrum of Eq. (6). This more mathematical identification of the transitions and its relation to the physics described here is discussed in Appendix C.

\section{OBSERVING THE TRANSITIONS EXPERIMENTALLY}

The above physics can be readily observed in the setup of recent experiments [26] by adjusting the measurement strength/Rabi frequency. The simplest transition to observe is that at $\lambda=2$, which is apparent in routine experiments measuring the average polarization or survival probability in $|0\rangle$. Observing the transitions at $\lambda=1$ and $2 / \sqrt{3}$ requires sampling the distribution $P_{\infty}(\theta)$ by tracking individual quantum trajectories for sufficiently long times and performing quantum tomography on the final states. This is possible with state-of-the-art experimental techniques [5,26], though laborious. A somewhat less laborious alternative to observe the $\lambda=1$ transition is to measure the probability to observe no clicks for a given time, $P^{(0)}(t)$. While this requires recording every measurement outcome, it does not require knowing the qubit state at time $t$. For the transition at $\lambda=2 / \sqrt{3}$, one can measure the probability to reach a specific $\theta$ by a sequence of no clicks, $P^{(0)}(\theta)$, which requires further tracing the qubit state up to time $t$. This can be done either by inferring the state from the theoretical dependence $\theta(t)$ or via a tomography of states postselected on $r=0$ readouts (which has been implemented in Ref. [26] for $\lambda \gg 1$ ).

\section{CONCLUSIONS}

Here we have studied the full stochastic dynamics of a system subject to a constant Hamiltonian and a continuous partial measurement. We have shown that the onset of the Zeno-like regime is preceded by a number of drastic qualitative changes in the system dynamics. Each such transition introduces a different feature of the fully localized dynamics, starting with the opening up of a finite-size region of forbidden states, followed by a singularity in the steady-state probability distribution of states, and ultimately a nonoscillatory dynamics of the qubit survival probability. We have proposed how to observe our findings in current experiments. Strikingly, depending on the definition of the "Zeno-like regime," one could call each of the transitions its onset. For example, the probability of observing a long sequence of no clicks starts increasing with increasing the measurement strength at $\lambda=1$. The survival probability starts increasing with increasing the measurement strength only after the last transition at $\lambda=2$. Some of our findings may depend on the specific measurement model, making it of interest to study the onset of the Zeno regime beyond continuous partial measurement. 


\section{ACKNOWLEDGMENTS}

We thank Serge Rosenblum and Fabien Lafont for their comments on the paper. K.S. and P.K. acknowledge funding by the Deutsche Forschungsgemeinschaft Grants No. 277101999 (TRR 183 Project No. C01) and No. EG 96/13-1, as well as by the Israel Science Foundation. A.R. acknowledges Engineering and Physical Sciences Research Council (GB) Grant No. EP/P010180/1.

K.S. and P.K. contributed equally to this work.

\section{APPENDIX A: A PHYSICAL MODEL OF THE MEASUREMENT}

The system under consideration in the paper is a qubit $(|0\rangle$, $|1\rangle)$ evolving under its own Hamiltonian and being measured by a two-state detector $\left(\left|0_{d}\right\rangle,\left|1_{d}\right\rangle\right)$ at intervals $d t$. The system's Hamiltonian is

$$
H_{s}=\Omega_{s} \sigma_{x}^{(s)} .
$$

We consider a system-detector Hamiltonian given by

$$
H_{s-d}=\frac{J}{2}\left(1-\sigma_{z}^{(s)}\right) \sigma_{y}^{(d)},
$$

where the detector is also assumed to be a two-level system. The detector is initially prepared in the state $\left|0_{d}\right\rangle$ for each measurement, i.e., at the beginning of each time step. The system's evolution under the combined effect of its Hamiltonian and the coupling to the detector is given by the unitary evolution due to

$$
H=H_{s}+H_{s-d},
$$

for time $d t$, after which the detector is read out with readouts $r=0,1$ corresponding to it being in $\left|r_{d}\right\rangle$.

We consider the scaling limit of continuous measurements defined as $d t \rightarrow 0, J^{2} d t \rightarrow \alpha=$ const (i.e., $J=\sqrt{\alpha / d t}$ ). In this limit, the measurement and the system evolution do not intermix in a single step; therefore,

$$
|\psi(t+d t)\rangle=M^{(r)} U_{s}|\psi(t)\rangle .
$$

The unitary evolution due to the system's Hamiltonian is

$$
\begin{aligned}
U_{s} & =e^{-i H_{s} d t}=\cos \Omega_{s} d t-i \sigma_{x}^{(s)} \sin \Omega_{s} d t \\
& =\left(\begin{array}{cc}
1 & -i \Omega_{s} d t \\
-i \Omega_{s} d t & 1
\end{array}\right)+O\left(d t^{2}\right) .
\end{aligned}
$$

The measurement back-action matrices, combining the effects of the system-detector evolution and the readout in the state $\left|r_{d}\right\rangle$, defined as

$$
M^{(r)}=\left\langle r_{d}\left|e^{-i H_{s-d} d t}\right| 0_{d}\right\rangle,
$$

are

$$
\begin{gathered}
M^{(0)}=\left(\begin{array}{cc}
1 & 0 \\
0 & \cos J d t
\end{array}\right)=\left(\begin{array}{cc}
1 & 0 \\
0 & 1-\frac{1}{2} \alpha d t
\end{array}\right)+O\left(d t^{2}\right), \\
M^{(1)}=\left(\begin{array}{cc}
0 & 0 \\
0 & \sin J d t
\end{array}\right)=\left(\begin{array}{cc}
0 & 0 \\
0 & \sqrt{\alpha d t}
\end{array}\right)+O\left(d t^{3 / 2}\right) .
\end{gathered}
$$

These are the operators in Eq. (1) of the paper describing the effect of the measurements.

\section{APPENDIX B: CALCULATION OF POSTSELECTION AND SURVIVAL PROBABILITIES}

In the main text we have presented some of the observable signatures of the transition in terms of the probabilities $P^{(0)}(t)$ to observe a no-click sequence of duration $t$ and $P^{(0)}(\theta)$ to reach $\theta$ by a sequence of no clicks, as well as the behavior of the average state polarization $\overline{\boldsymbol{s}}(t)$. Here we derive the results stated in the main text.

\section{Probabilities $P^{(0)}(t)$ and $P^{(0)}(\theta)$}

To determine the probability of the $r=0$ postselected trajectory, we start by solving the state evolution under a sequence of zero readouts. The corresponding equation for $\theta$ [see Eq. (3)] is

$$
\frac{d \theta}{d t}=-2 \Omega_{s}(1+\lambda \sin \theta)
$$

The solution is

$$
\tan \frac{\theta}{2}=\sqrt{\lambda^{2}-1} \tanh \left[\Omega_{s} \sqrt{\lambda^{2}-1}\left(t-t_{0}\right)\right]-\lambda .
$$

Setting the initial condition $\theta(t=0)=0$ and simplifying the expression, we arrive to

$$
\begin{aligned}
\tan \frac{\theta(t)}{2} & =\sqrt{\lambda^{2}-1} \operatorname{coth}\left(\Omega_{s} t \sqrt{\lambda^{2}-1}-\frac{1}{2} \ln \frac{\tan \frac{\theta_{+}}{2}}{\tan \frac{\theta_{-}}{2}}\right)-\lambda \\
& =-\frac{1}{\lambda+\sqrt{\lambda^{2}-1} \operatorname{coth}\left(\Omega_{s} t \sqrt{\lambda^{2}-1}\right)} .
\end{aligned}
$$

For $\lambda>1$, this expression describes the evolution of $\theta(t)$ from $\pi$ at $t=-\frac{1}{\Omega_{s} \sqrt{\lambda^{2}-1}} \operatorname{arccoth}\left(\frac{\lambda}{\sqrt{\lambda^{2}-1}}\right)$ to $\theta_{+}$at $t=+\infty$. For $\lambda<$ 1, Eq. (B3) becomes

$$
\tan \frac{\theta(t)}{2}=-\frac{1}{\lambda+\sqrt{1-\lambda^{2}} \cot \left(\Omega_{s} t \sqrt{1-\lambda^{2}}\right)}
$$

and describes the periodic evolution of $\theta(t)$ with period $T=$ $\frac{\pi}{\Omega_{s} \sqrt{\lambda^{2}-1}}$.

We are interested in the probability of having zero readout at time $t, P_{0}(t)$. Knowing the probability of obtaining $r=0$ in each infinitesimal step, the equation for $P_{0}(t)$ is readily determined:

$$
\begin{aligned}
\frac{d P^{(0)}(t)}{d t} & =-\frac{p_{1}}{d t} P^{(0)}(t)=-\alpha \sin ^{2} \frac{\theta(t)}{2} P^{(0)}(t)=-\alpha \frac{\tan ^{2} \frac{\theta(t)}{2}}{1+\tan ^{2} \frac{\theta(t)}{2}} P^{(0)}(t) \\
& =-\alpha \frac{\sinh ^{2}\left(\Omega_{s} t \sqrt{\lambda^{2}-1}\right)}{\lambda^{2} \cosh \left(2 \Omega_{s} t \sqrt{\lambda^{2}-1}\right)-1+\lambda \sqrt{\lambda^{2}-1} \sinh \left(2 \Omega_{s} t \sqrt{\lambda^{2}-1}\right)} .
\end{aligned}
$$


Integrating the equation and demanding $P_{0}(t=0)=1$, one obtains

$$
P^{(0)}(t)=e^{-2 \Omega_{s} \lambda t} \frac{\lambda^{2} \cosh \left(2 \Omega_{s} t \sqrt{\lambda^{2}-1}\right)-1+\lambda \sqrt{\lambda^{2}-1} \sinh \left(2 \Omega_{s} t \sqrt{\lambda^{2}-1}\right)}{\lambda^{2}-1} .
$$

This expression is not singular at $\lambda=1$, where it becomes $P_{0}(t)=\left(1+2 \Omega_{s} t+2 \Omega_{s}^{2} t^{2}\right) e^{-2 \Omega_{s} t}$. For $\lambda<1$, it reduces to

$$
P^{(0)}(t)=e^{-2 \Omega_{s} \lambda t} \frac{\lambda^{2} \cos \left(2 \Omega_{s} t \sqrt{1-\lambda^{2}}\right)-1-\lambda \sqrt{1-\lambda^{2}} \sin \left(2 \Omega_{s} t \sqrt{1-\lambda^{2}}\right)}{\lambda^{2}-1} .
$$

From Eq. (B7), one can directly derive the long-time behavior of $P^{(0)}(t)$ reported in the paper [see Eq. (10)], which is

$$
P^{(0)}(t \rightarrow+\infty) \propto \exp \left(-2 \Omega_{s} \lambda t\right) \times \text { oscillating function, }
$$

for $\lambda<1$, and

$$
P^{(0)}(t \rightarrow+\infty) \propto \exp \left(-2 \Omega_{s}\left[\lambda-\sqrt{\lambda^{2}-1}\right] t\right)
$$

for $\lambda>1$

The probability to reach state $\theta$ via a sequence of $r=0$ readouts, $P^{(0)}(\theta)$, is obtained from the equation

$$
\frac{d P_{0}(\theta)}{d \theta}=\frac{d P_{0}[\theta(t)]}{d t} / \frac{d \theta}{d t}=\frac{2 \lambda \sin ^{2} \frac{\theta}{2}}{1+\lambda \sin \theta} P_{0}(\theta) .
$$

The solution with $P^{(0)}(\theta=0)=1$ is

$$
\begin{aligned}
P^{(0)}(\theta) & =\frac{1}{1+\lambda \sin \theta}\left(\frac{\tan \frac{\theta}{2}-\tan \frac{\theta_{+}}{2}}{\tan \frac{\theta}{2}-\tan \frac{\theta_{-}}{2}}\right)^{\frac{\lambda}{\sqrt{\lambda^{2}-1}}}\left(\frac{\tan \frac{\theta_{+}}{2}}{\tan \frac{\theta_{-}}{2}}\right)^{-\frac{\lambda}{\sqrt{\lambda^{2}-1}}} \\
& =\frac{1}{1+\lambda \sin \theta} \exp \left(\frac{2 \lambda}{\sqrt{1-\lambda^{2}}}\left[\arctan \frac{\lambda+\tan \frac{\theta}{2}}{\sqrt{1-\lambda^{2}}}-\arctan \frac{\lambda}{\sqrt{1-\lambda^{2}}}\right]\right) .
\end{aligned}
$$

When $\lambda>1$, starting at $\theta=0$ it is possible to reach only the states with $\theta \in\left(\theta_{+} ; 0\right]$, which is reflected in the vanishing of

$$
P^{(0)}\left(\theta \approx \theta_{+}\right) \propto\left(\tan \frac{\theta}{2}-\tan \frac{\theta_{+}}{2}\right)^{\frac{\lambda}{\sqrt{\lambda^{2}-1}}-1},
$$

and in the properties of its derivatives discussed in the paper.

\section{Survival probability and the average state polarization}

The final quantity used in the paper to describe the dynamics of the system is the average state polarization after time $t, \bar{s}(t) \equiv\left(\bar{s}_{y}(t), \bar{s}_{z}(t)\right)$, which is related to the survival probability in the initial state $|0\rangle, \mathcal{P}(t)=\left[1+\bar{s}_{z}(t)\right] / 2$, i.e., the probability to measure the system in $|0\rangle$ upon a projective measurement at time $t$. These quantities require averaging over all the state trajectories, and no postselection is required. The average $\bar{s}_{y}$ and $\bar{s}_{z}$ components of the polarization can be expressed through the state distribution $P_{t}(\theta)$ :

$$
\begin{aligned}
& \bar{s}_{y}(t)=\int_{-\pi}^{\pi} d \theta P_{t}(\theta) \sin \theta, \\
& \bar{s}_{z}(t)=\int_{-\pi}^{\pi} d \theta P_{t}(\theta) \cos \theta .
\end{aligned}
$$

It then follows from Eq. (6) that

$$
\frac{d}{d t}\left(\begin{array}{c}
\bar{s}_{y} \\
\bar{s}_{z}
\end{array}\right)=\left(\begin{array}{cc}
-\frac{\alpha}{2} & -2 \Omega_{s} \\
2 \Omega_{s} & 0
\end{array}\right)\left(\begin{array}{c}
\bar{s}_{y} \\
\bar{s}_{z}
\end{array}\right)
$$

The evolution has two eigenvalues, $\Omega_{s}\left(-\lambda \pm \sqrt{\lambda^{2}-4}\right)$. When the system is initialized in state $|0\rangle$ at $t=0$, the evolution of $\overline{\boldsymbol{s}}(t)$ is given by

$$
\begin{gathered}
\bar{s}_{y}(t)=-2 e^{-\Omega_{s} \lambda t} \frac{\sinh \Omega_{s} t \sqrt{\lambda^{2}-4}}{\sqrt{\lambda^{2}-4}}, \\
\bar{s}_{z}(t)=e^{-\Omega_{s} \lambda t}\left(\cosh \Omega_{s} t \sqrt{\lambda^{2}-4}+\frac{\lambda}{\sqrt{\lambda^{2}-4}} \sinh \Omega_{s} t \sqrt{\lambda^{2}-4}\right) .
\end{gathered}
$$

Its long-time behavior is given by

$$
\bar{s}_{y, z}(t) \propto \begin{cases}\exp \left(-\Omega_{s} \lambda t\right) \times \text { oscillating function, } & \text { for } \lambda<2, \\ \exp \left(-\Omega_{s}\left[\lambda-\sqrt{\lambda^{2}-4}\right] t\right), & \text { for } \lambda>2 .\end{cases}
$$

Therefore, at long times, the survival probability $\mathcal{P}(t)$ decays to the steady-state value $\mathcal{P}(t \rightarrow \infty)=1 / 2$. The decay rate is $\Omega_{s}\left(\lambda-\sqrt{\lambda^{2}-4}\right)=2 \Omega_{s}\left(\frac{\lambda-\sqrt{\lambda^{2}-4}}{2}\right)$. It exhibits a maximum at $\lambda=2$, resembling the maximum of the decay rate of $P_{0}(t)$ at $\lambda=1$.

Note that the equation for the polarization evolution, yielding two eigenvalues, $\Omega_{s}\left(\lambda \pm \sqrt{\lambda^{2}-4}\right)$, has been obtained from Eq. (6). Thus, Eq. (6) "knows" about the eigenvalues $\gamma=\frac{1}{2}\left(-\lambda \pm \sqrt{\lambda^{2}-4}\right)$ for any values of $\lambda$. At the same time, these eigenvalues correspond to normalizable eigenmodes of Eq. (6) only when $\lambda<2 / \sqrt{3}$ (see the discussion in Appendix $\mathrm{C} 3$ ). 


\section{APPENDIX C: DERIVATION AND THE EIGENSPECTRUM OF THE MASTER EQUATION}

\section{Derivation of Eq. (6)}

The stochastic dynamics of the system is described by the probability density $P_{t}(\theta)$ of being in the state $|\psi(\theta)\rangle$ at time $t$ for the stochastic variable $\theta$. The probability of the system being in an interval of states $\left[\theta_{1}, \theta_{2}\right]$ at time $t, P_{t}\left(\left[\theta_{1} ; \theta_{2}\right]\right)=$ $\int_{\theta_{1}}^{\theta_{2}} d \theta P_{t}(\theta)$, obeys the evolution

$$
\begin{aligned}
\int_{\theta_{1}}^{\theta_{2}} d \theta P_{t+d t}(\theta)= & \int_{\tilde{\theta}_{1}}^{\tilde{\theta}_{2}} d \tilde{\theta} P_{t}(\tilde{\theta}) p_{0}(\tilde{\theta})+\Theta_{H}\left(\pi \in\left[\theta_{1} ; \theta_{2}\right]\right) \\
& \times \int_{0}^{2 \pi} d \tilde{\theta} p_{1}(\tilde{\theta}) P_{t}(\tilde{\theta}),
\end{aligned}
$$

where $\Theta_{H}(x \in[a ; b])$ is 1 if $x \in[a, b]$ and zero otherwise, and $p_{r}(\theta)$ are the probabilities of obtaining the readout $r$ in a measurement:

$$
p_{r=1} \equiv p_{1}=\alpha d t \sin ^{2} \frac{\theta(t)}{2}, \quad p_{r=0} \equiv p_{0}=1-p_{1} .
$$

The first term on the right-hand side of Eq. (C1) describes the change of $P_{t+d t}\left(\left[\theta_{1}^{\prime} ; \theta_{2}^{\prime}\right]\right)$ due to the the smooth evolution under $r=0$ readout, while the second term accounts for jumps to $\theta=\pi$ for the measurement outcome $r=1$. The variables $\tilde{\theta}_{1,2}$ are defined via the self-consistent condition $\tilde{\theta}_{1,2}-\Omega\left(\tilde{\theta}_{1,2}\right) d t=\theta_{1,2}$, where $\Omega(\theta)=2 \Omega_{s}[1+\lambda \sin \theta$ ] [see Eq. (3)]. A differential equation for $P_{t}(\theta)$ is obtained by solving the self-consistent equation to order $(d t)^{2}$, differentiating Eq. (C1) over $\theta_{2}$, and retaining the terms of order $d t$. With the explicit expressions for $p_{r}$ and $\Omega(\theta)$, we get Eq. (6).

The integrodifferential master equation (6) needs to be supplemented by the boundary conditions. They are simple:

$$
P_{t}(\theta=0)=P_{t}(\theta=2 \pi) .
$$

In other words, $P_{t}(\theta)=P_{t}(\theta+2 \pi)$. With this condition, it is easy to check that the normalization by total probability $\int_{0}^{2 \pi} d \theta P_{t}(\theta)=1$ is preserved by this equation. Finally, it is useful for solving to eliminate the integral part of the equation. This is easily done, as it only contributes at $\theta=\pi$. Therefore, Eq. (6) is equivalent to

$$
\frac{d P_{t}(\theta)}{d t}=-\alpha \sin ^{2} \frac{\theta}{2} P_{t}(\theta)+\partial_{\theta}\left[\left(2 \Omega_{s}+\frac{\alpha}{2} \sin \theta\right) P_{t}(\theta)\right]
$$

at $\theta \neq \pi$, supplemented with boundary condition

$$
2 \Omega_{s}\left[P_{t}(\pi+0)-P_{t}(\pi-0)\right]=-\alpha \int_{0}^{2 \pi} d \theta \sin ^{2} \frac{\theta}{2} P_{t}(\theta) .
$$

\section{Eigenmodes of Eq. (6)}

We now derive the eigenmode solutions of Eq. (6), i.e., find all the solutions of the form $P_{t}(\theta)=e^{2 \Omega_{s} \gamma t} f_{\gamma}(\theta)$. The reader may skip the details and look at the result in Appendix C3.

Before diving into the derivation, it is useful to analyze the expectations from the solution. Due to the normalization condition, for every eigenmode with $\gamma \neq 0$, we should have $\int_{0}^{2 \pi} d \theta f_{\gamma}(\theta)=0$. Since $P_{t}(\theta) \geqslant 0$, there can be no eigenmodes with $\gamma>0$. Thus, only solutions with $\gamma \leqslant 0$ are acceptable. Finally, since the decay rate of clicks at $\theta_{+}$ is $p_{1}(\theta) / d t=\alpha \sin ^{2} \frac{\theta_{+}}{2}$, for $\lambda>1$ we expect an eigenmode with $2 \Omega_{s} \gamma \geqslant-\alpha \sin ^{2} \frac{\theta_{+}}{2}=-\alpha \tan ^{2} \frac{\theta_{+}}{2} /\left(1+\tan ^{2} \frac{\theta_{+}}{2}\right)=$ $2 \Omega_{s}\left(\sqrt{\lambda^{2}-1}-\lambda\right)$, i.e., $\gamma \geqslant \sqrt{\lambda^{2}-1}-\lambda$. We also expect a steady-state solution with $\gamma=0$ to exist.

\section{a. The functional dependence}

Away from $\theta=\pi$, the equation for the eigenmodes is

$$
-\alpha \sin ^{2} \frac{\theta}{2} f_{\gamma}(\theta)+\partial_{\theta}\left[\left(2 \Omega_{s}+\frac{\alpha}{2} \sin \theta\right) f_{\gamma}(\theta)\right]=2 \Omega_{s} \gamma f_{\gamma}(\theta) .
$$

Equivalently,

$$
\left(2 \Omega_{s}+\frac{\alpha}{2} \sin \theta\right) f_{\gamma}^{\prime}(\theta)=\left[\frac{\alpha}{2}(1-2 \cos \theta)+2 \Omega_{s} \gamma\right] f_{\gamma}(\theta)
$$

Note that for $\alpha \geqslant 4 \Omega_{s}$, at $\theta=\theta_{ \pm}$, the equation becomes singular as the factor multiplying the highest (and only) derivative vanishes. These singular points require special treatment. However, this simply means that $f_{\gamma}\left(\theta \in\left[-\pi ; \theta_{+}\right]\right)=0$ as this interval is inaccessible from the time evolution of any state initially outside it, as shown in the analysis of the postselected dynamics in the paper. We will come back to the issue of the special points later.

Away from the special points, the equation admits an analytic solution, which can be expressed in two alternative forms:

$$
f_{\gamma}(\theta)=\frac{C}{(1+\lambda \sin \theta)^{2}} \exp \left(2 \frac{\lambda+\gamma}{\sqrt{1-\lambda^{2}}}\left[\arctan \frac{\lambda+\tan \frac{\theta}{2}}{\sqrt{1-\lambda^{2}}}-\frac{\pi}{2}\right]\right)=\frac{C}{(1+\lambda \sin \theta)^{2}}\left(\frac{\tan \frac{\theta}{2}-\tan \frac{\theta_{+}}{2}}{\tan \frac{\theta}{2}-\tan \frac{\theta_{-}}{2}}\right)^{\frac{\lambda+\gamma}{\sqrt{\lambda^{2}-1}}},
$$

where $\lambda=\alpha /\left(4 \Omega_{s}\right)$. The equivalence of the two expressions follows from

$$
\arctan x=\frac{1}{2 i} \ln \frac{1+i x}{1-i x},
$$

so one can write

$$
\left(\frac{\tan \frac{\theta}{2}-\tan \frac{\theta_{+}}{2}}{\tan \frac{\theta}{2}-\tan \frac{\theta_{-}}{2}}\right)^{y}=\left(\frac{\tan \frac{\theta}{2}+\lambda-\sqrt{\lambda^{2}-1}}{\tan \frac{\theta}{2}+\lambda+\sqrt{\lambda^{2}-1}}\right)^{y}=\exp \left[2 y\left(\arctan \frac{\lambda+\tan \frac{\theta}{2}}{\sqrt{1-\lambda^{2}}}-\frac{\pi}{2}\right)\right] .
$$


Obviously, the first form in Eq. (C8) is more convenient for $\lambda<1$, while the second one is the natural choice for $\lambda>1$. However, for computational purposes one can use either. Note the singularities at $\theta=\theta_{+}$and $\theta_{-}$for $\lambda>1$ (for $\lambda<1$, $\tan \frac{\theta_{ \pm}}{2}-\tan \frac{\theta}{2} \neq 0$ for any $\theta$ since $\tan \frac{\theta_{ \pm}}{2}$ has a nonzero imaginary part while $\tan \frac{\theta}{2}$ is a real function). From the equality

$$
1+\lambda \sin \theta=\frac{1+2 \lambda \tan \frac{\theta}{2}+\tan ^{2} \frac{\theta}{2}}{1+\tan ^{2} \frac{\theta}{2}}=\frac{\left(\tan \frac{\theta}{2}-\tan \frac{\theta_{+}}{2}\right)\left(\tan \frac{\theta}{2}-\tan \frac{\theta_{-}}{2}\right)}{1+\tan ^{2} \frac{\theta}{2}},
$$

we obtain the following behavior in proximity of the singularity points at $\theta=\theta_{ \pm}$:

$$
\begin{aligned}
& f\left(\theta \approx \theta_{+}\right) \sim\left(\tan \frac{\theta}{2}-\tan \frac{\theta_{+}}{2}\right)^{\frac{\lambda+\gamma}{\sqrt{\lambda^{2}-1}}-2}, \\
& f\left(\theta \approx \theta_{-}\right) \sim\left(\tan \frac{\theta}{2}-\tan \frac{\theta_{-}}{2}\right)^{-\frac{\lambda+\gamma}{\sqrt{\lambda^{2}-1}}-2} .
\end{aligned}
$$

The normalizability conditions

$$
\left|\int^{\theta_{+}} d \theta f(\theta)\right|<\infty, \quad\left|\int^{\theta_{-}} d \theta f(\theta)\right|<\infty
$$

for Eqs. (C11) and (C12) imply that, for $\lambda>1$, one must have

$$
\begin{aligned}
& \frac{\lambda+\operatorname{Re} \gamma}{\sqrt{\lambda^{2}-1}}>1\left(\text { vicinity of } \theta_{+}\right), \\
& \frac{\lambda+\operatorname{Re} \gamma}{\sqrt{\lambda^{2}-1}}<-1\left(\text { vicinity of } \theta_{-}\right) .
\end{aligned}
$$

These two conditions are incompatible. The apparent contradiction is resolved by choosing the normalization constant $C$ in Eq. (C8) independently on intervals $\left(-\pi ; \theta_{-}\right),\left(\theta_{-} ; \theta_{+}\right)$, and $\left(\theta_{+} ; \pi\right)$. Then, for normalizable solutions, $C=0$ either on the first two intervals or on the last two intervals. We do not investigate the case of $C \neq 0$ on $\left(-\pi ; \theta_{-}\right)$, as these solutions (if exist) describe quick escape from the interval and cannot contribute if the system is initialized outside of it. Choosing $C=0$ in the first two intervals, the eigenmodes are normalizable, and one recovers the expected property $f_{\gamma}\left(\theta \in\left[-\pi ; \theta_{+}\right]\right)=0$.

Putting these results together, we have the general expression for the eigenmodes, which reads, for $\lambda>1$,

$$
f_{\gamma}(\theta)= \begin{cases}\frac{C\left(1+\tan ^{2} \frac{\theta}{2}\right)^{2}}{\left(\tan \frac{\theta}{2}-\tan \frac{\theta_{+}}{2}\right)^{2}\left(\tan \frac{\theta}{2}-\tan \frac{\theta_{-}}{2}\right)^{2}}\left(\frac{\tan \frac{\theta}{2}-\tan \frac{\theta_{+}}{2}}{\tan \frac{\theta}{2}-\tan \frac{\theta_{-}}{2}}\right)^{\frac{\lambda+\gamma}{\sqrt{\lambda^{2}-1}}}, & \text { for } \theta \in\left[\theta_{+} ; \pi\right], \\ 0, & \text { for } \theta \in\left[-\pi ; \theta_{+}\right],\end{cases}
$$

and, for $\lambda<1$,

$$
f_{\gamma}(\theta)=\frac{C}{(1+\lambda \sin \theta)^{2}} \exp \left(2 \frac{\lambda+\gamma}{\sqrt{1-\lambda^{2}}}\left[\arctan \frac{\lambda+\tan \frac{\theta}{2}}{\sqrt{1-\lambda^{2}}}-\frac{\pi}{2}\right]\right), \quad \text { for } \theta \in(-\pi ; \pi)
$$

\section{b. The boundary conditions and normalization}

The above does not present the final solution. We have two boundary conditions and a normalization condition to satisfy. The first boundary condition, $f_{\gamma}(\theta)=f_{\gamma}(\theta+2 \pi)$, is satisfied trivially.

The second boundary condition, which needs to be addressed further, Eq. (C5), yields

$$
2 \Omega_{s}\left[f_{\gamma}(\pi-0)-f_{\gamma}(\pi+0)\right]=\alpha \int_{-\pi}^{\pi} d \theta \sin ^{2} \frac{\theta}{2} f_{\gamma}(\theta) .
$$

Note that this condition is independent of $C$, hence it determines the spectrum of eigenmodes $\gamma$.

The normalization condition is

$$
\int_{0}^{2 \pi} d \theta f_{\gamma}(\theta)= \begin{cases}1, & \text { for } \gamma=0 \\ 0, & \text { for } \gamma \neq 0\end{cases}
$$

This should be used to determine $C$ for the steady state $(\gamma=0)$ and should be satisfied automatically for all $\gamma \neq 0$ eigenmodes. The integrals can be calculated analytically:

$$
\begin{aligned}
\int d \theta f(\theta) & =2 C \int d t \frac{1+t^{2}}{\left(t-\tan \frac{\theta_{+}}{2}\right)^{2}\left(t-\tan \frac{\theta_{-}}{2}\right)^{2}}\left(\frac{t-\tan \frac{\theta_{+}}{2}}{t-\tan \frac{\theta_{-}}{2}}\right)^{\frac{\lambda+\gamma}{\sqrt{\lambda^{2}-1}}} \\
& =\frac{C}{\lambda+\gamma}\left(\frac{t-\tan \frac{\theta_{+}}{2}}{t-\tan \frac{\theta_{-}}{2}}\right)^{\frac{\lambda+\gamma}{\sqrt{\lambda^{2}-1}}}\left(1+\frac{\lambda\left[(2 \lambda+\gamma)(1-2 \gamma t)-\gamma t^{2}\right]}{\left(t-\tan \frac{\theta_{+}}{2}\right)\left(t-\tan \frac{\theta_{-}}{2}\right)\left(\gamma-\tan \frac{\theta_{+}}{2}\right)\left(\gamma-\tan \frac{\theta_{-}}{2}\right)}\right),
\end{aligned}
$$


where $t=\tan \frac{\theta}{2}$. Similarly,

$$
\begin{aligned}
\int d \theta \sin ^{2} \frac{\theta}{2} f(\theta) & =2 C \int d t \frac{t^{2}}{\left(t-\tan \frac{\theta_{+}}{2}\right)^{2}\left(t-\tan \frac{\theta_{-}}{2}\right)^{2}}\left(\frac{t-\tan \frac{\theta_{+}}{2}}{t-\tan \frac{\theta_{-}}{2}}\right)^{\frac{\lambda+\gamma}{\sqrt{\lambda^{2}-1}}} \\
& =\frac{C}{2(\lambda+\gamma)}\left(t^{2}+\frac{(\gamma t-1)^{2}}{\left(\gamma-\tan \frac{\theta_{+}}{2}\right)\left(\gamma-\tan \frac{\theta_{-}}{2}\right)}\right) \frac{1}{\left(t-\tan \frac{\theta_{+}}{2}\right)\left(t-\tan \frac{\theta_{-}}{2}\right)}\left(\frac{t-\tan \frac{\theta_{+}}{2}}{t-\tan \frac{\theta_{-}}{2}}\right)^{\frac{\lambda+\gamma}{\sqrt{\lambda^{2}-1}}} .
\end{aligned}
$$

c. The solution for $\lambda>1$ and $\theta \in\left[\theta_{+} ; \pi\right]$

Assuming $\frac{\lambda+\operatorname{Re} \gamma}{\sqrt{\lambda^{2}-1}}>1$, the boundary condition yields

$$
2 \Omega_{s} C=\alpha \int_{\theta_{+}}^{\pi} d \theta \sin ^{2} \frac{\theta}{2} f_{\gamma}(\theta)=\frac{\alpha C\left(2 \gamma^{2}+2 \lambda \gamma+1\right)}{2(\lambda+\gamma)\left(\gamma^{2}+2 \lambda \gamma+1\right)} \Longleftrightarrow C \gamma\left(\gamma^{2}+\gamma \lambda+1\right)=0 .
$$

This fixes the possible $\gamma$, thus giving us three eigenmodes:

$$
\gamma=0, \gamma=\frac{1}{2}\left(-\lambda \pm \sqrt{\lambda^{2}-4}\right)
$$

The norm of the eigenmodes is then

$$
\int_{\theta_{+}}^{\pi} d \theta f(\theta)=\frac{C}{\lambda+\gamma}\left(1-\frac{\gamma \lambda}{\left(\gamma^{2}+2 \lambda \gamma+1\right)}\right)=\frac{C}{\lambda+\gamma} \frac{\gamma^{2}+\gamma \lambda+1}{\gamma^{2}+2 \lambda \gamma+1}
$$

which implies that the eigenfunctions with $\gamma=\frac{1}{2}\left(-\lambda \pm \sqrt{\lambda^{2}-4}\right)$ integrate to zero as expected. For the steady state, $\gamma=0$, the normalization condition $\int_{\theta_{+}}^{\pi} d \theta f(\theta)=1$ yields

$$
\begin{gathered}
C=\lambda, \\
f_{\gamma=0}(\theta)=\frac{\lambda\left(1+\tan ^{2} \frac{\theta}{2}\right)^{2}}{\left(\tan \frac{\theta}{2}-\tan \frac{\theta_{+}}{2}\right)^{2}\left(\tan \frac{\theta}{2}-\tan \frac{\theta_{-}}{2}\right)^{2}}\left(\frac{\tan \frac{\theta}{2}-\tan \frac{\theta_{+}}{2}}{\tan \frac{\theta}{2}-\tan \frac{\theta_{-}}{2}}\right)^{1 / \cos \theta_{+}} \\
=\lambda \frac{\left(1+\tan ^{2} \frac{\theta}{2}\right)^{2}}{\left(\tan ^{2} \frac{\theta}{2}+2 \lambda \tan \frac{\theta}{2}+1\right)^{2}}\left(\frac{\tan \frac{\theta}{2}+\lambda-\sqrt{\lambda^{2}-1}}{\tan \frac{\theta}{2}+\lambda+\sqrt{\lambda^{2}-1}}\right)^{\lambda / \sqrt{\lambda^{2}-1}} .
\end{gathered}
$$

Finally, we check the conditions in Eqs. (C11) and (C12):

$$
\frac{\lambda+\operatorname{Re} \gamma}{\sqrt{\lambda^{2}-1}}>1 \Longleftrightarrow \operatorname{Re} \gamma>\sqrt{\lambda^{2}-1}-\lambda .
$$

Interestingly, the condition actually requires that all the eigenmodes decay not slower than the decay rate at $\theta=\theta_{+}$. The steady state, $\gamma=0$, always satisfies the condition for $\lambda \in$ $(1 ;+\infty)$. For $\lambda \leqslant 2, \operatorname{Re} \frac{1}{2}\left(-\lambda \pm \sqrt{\lambda^{2}-4}\right)=-\lambda / 2$. Then, the normalizability condition is equivalent to

$$
\lambda>2 \sqrt{\lambda^{2}-1} \Leftrightarrow 3 \lambda^{2}<4 \Leftrightarrow \lambda<2 / \sqrt{3} .
$$

For $\quad \lambda>2, \quad$ the inequality $\frac{1}{2}\left(-\lambda-\sqrt{\lambda^{2}-4}\right)<$ $\frac{1}{2}\left(-\lambda+\sqrt{\lambda^{2}-4}\right)<\sqrt{\lambda^{2}-1}-\lambda$ always holds. Therefore, the eigenmodes $\gamma=\frac{1}{2}\left(-\lambda \pm \sqrt{\lambda^{2}-4}\right)$ are only normalizable for $\lambda<2 / \sqrt{3}$.

\section{d. The solution for $\lambda<1$}

The same considerations hold for $0 \leqslant \lambda<1$. In this range of parameters, $\sqrt{\lambda^{2}-1}$ and $\tan \frac{\theta_{ \pm}}{2}$ become complex. Therefore, it is more convenient to use $f_{\gamma}(\theta)$ in the first form in Eq. (C8). At the same time, the integrals for the norm and the boundary condition at $\theta=\pi$ are more conveniently calculated using the expressions in Eq. (C16) (see Appendix $\mathrm{C} 2 \mathrm{~b}$ ). Using these expressions and the results of Appendix $\mathrm{C} 2 \mathrm{~b}$, one finds that the boundary condition at $\theta=\pi$ yields

$\frac{C}{\lambda+\gamma} \frac{\gamma^{2}+\gamma \lambda+1}{\gamma^{2}+2 \lambda \gamma+1}\left(1-\exp \left[-2 \pi \frac{\lambda+\gamma}{\sqrt{1-\lambda^{2}}}\right]\right)=0$.

Therefore, on top of the three eigenmodes, $\gamma=0$ and $\frac{1}{2}\left(-\lambda \pm \sqrt{\lambda^{2}-4}\right)$, there is also an infinite sequence of eigenvalues given by

$$
\gamma=-\lambda+i m \sqrt{1-\lambda^{2}}, \quad m \in \mathbb{Z} .
$$

Since there are no special points for $\lambda<1$, both this infinite set of solutions and the three eigenmodes in Eq. (C23) correspond to valid normalizable eigenmodes. 
For the steady state, the normalization $\int_{-\pi}^{\pi} d \theta f(\theta)=1$ yields

$$
\begin{gathered}
C=\frac{\lambda}{1-\exp \left(-\frac{2 \pi \lambda}{\sqrt{1-\lambda^{2}}}\right)}, \\
f_{\gamma=0}(\theta)=\frac{\lambda}{1-\exp \left(-\frac{2 \pi \lambda}{\sqrt{1-\lambda^{2}}}\right)} \frac{1}{(1+\lambda \sin \theta)^{2}} \\
\times \exp \left[\frac{2 \lambda}{\sqrt{1-\lambda^{2}}}\left(\arctan \frac{\lambda+\tan \frac{\theta}{2}}{\sqrt{1-\lambda^{2}}}-\frac{\pi}{2}\right)\right] .
\end{gathered}
$$

For $\gamma \neq 0$,

$$
\begin{aligned}
\int_{-\pi}^{\pi} d \theta f(\theta)= & \frac{C}{\lambda+\gamma} \frac{\gamma^{2}+\gamma \lambda+1}{\gamma^{2}+2 \lambda \gamma+1} \\
& \times\left(1-\exp \left[-2 \pi \frac{\lambda+\gamma}{\sqrt{1-\lambda^{2}}}\right]\right),
\end{aligned}
$$

which vanishes due to the boundary condition above, as expected.

\section{Summary of eigenvalues and eigenmodes of Eq. (6)}

Putting together the results from Appendix C2, we can summarize the solutions of the master equation (6) as follows. The steady state, with eigenvalue $\gamma=0$, is given for $\lambda<1$ by

$$
f_{0}(\theta)=\frac{\lambda}{1-\exp \left(-\frac{2 \pi \lambda}{\sqrt{1-\lambda^{2}}}\right)} \frac{1}{(1+\lambda \sin \theta)^{2}} \exp \left[\frac{2 \lambda}{\sqrt{1-\lambda^{2}}}\left(\arctan \frac{\lambda+\tan \frac{\theta}{2}}{\sqrt{1-\lambda^{2}}}-\frac{\pi}{2}\right)\right],
$$

while for $\lambda>1$ it reads

$$
f_{0}(\theta)= \begin{cases}\frac{\lambda}{(1+\lambda \sin \theta)^{2}}\left(\frac{\tan \frac{\theta}{2}+\lambda-\sqrt{\lambda^{2}-1}}{\tan \frac{\theta}{2}+\lambda+\sqrt{\lambda^{2}-1}}\right)^{\lambda / \sqrt{\lambda^{2}-1}}, & \text { for } \theta \in\left[\theta_{+} ; \pi\right], \\ 0, & \text { for } \theta \in\left[-\pi ; \theta_{+}\right],\end{cases}
$$

with $\tan \frac{\theta_{ \pm}}{2}=-\lambda \pm \sqrt{\lambda^{2}-1}, \lambda=\alpha /\left(4 \Omega_{s}\right)$, and

$$
\left(\frac{\tan \frac{\theta}{2}+\lambda-\sqrt{\lambda^{2}-1}}{\tan \frac{\theta}{2}+\lambda+\sqrt{\lambda^{2}-1}}\right)^{\lambda / \sqrt{\lambda^{2}-1}}=\exp \left[\frac{2 \lambda}{\sqrt{1-\lambda^{2}}}\left(\arctan \frac{\lambda+\tan \frac{\theta}{2}}{\sqrt{1-\lambda^{2}}}-\frac{\pi}{2}\right)\right] .
$$

These are the expressions used in the main text [see Eqs. (7) and (8)].

Several comments are in order. First, the expressions for both $\lambda<1$ and $\lambda>1$ are the same except for the normalization constant, which exhibits an essential singularity at $\lambda=1-\epsilon$. Second, exactly at the transition $\lambda=1$,

$$
f_{0}(\theta)= \begin{cases}\frac{1}{(1+\sin \theta)^{2}} \exp \left[-\frac{2}{1+\tan \frac{\theta}{2}}\right], & \theta \in[-\pi / 2 ; \pi], \\ 0, & \theta \in(-\pi ;-\pi / 2),\end{cases}
$$

which displays an essential singularity at $\theta=-\pi / 2$. Due to the nature of this singularity, the transition at $\lambda=1$ appears smooth when looking at the steady-state probability density. In this sense it could be regarded as a crossover.

The eigenmode spectrum consists of two sets of eigenvalues.

(1) $\gamma=-\lambda+i m \sqrt{1-\lambda^{2}}, \quad m \in \mathbb{Z}$ with

$$
f_{\gamma}(\theta)=\frac{C}{(1+\lambda \sin \theta)^{2}} \exp \left(2 \operatorname{im}\left[\arctan \frac{\lambda+\tan \frac{\theta}{2}}{\sqrt{1-\lambda^{2}}}-\frac{\pi}{2}\right]\right) .
$$

These eigenvalues exist for $\lambda \leqslant 1$, become massively degenerate at $\lambda=1$, and disappear at $\lambda>1$. This disappearance coincides with the opening of the forbidden region $\left(-\pi ; \theta_{+}\right)$. Note, thus, that the $\lambda=1$ transition, which appears as a crossover in the steady-state behavior, presents a drastic change in the eigenmode spectrum.

(2) $\gamma=\frac{1}{2}\left(-\lambda \pm \sqrt{\lambda^{2}-4}\right)$ with

$$
\begin{gathered}
f_{\gamma}(\theta)=\frac{C}{(1+\lambda \sin \theta)^{2}} \exp \left(\frac{\lambda \pm i \sqrt{4-\lambda^{2}}}{\sqrt{1-\lambda^{2}}}\left[\arctan \frac{\lambda+\tan \frac{\theta}{2}}{\sqrt{1-\lambda^{2}}}-\frac{\pi}{2}\right]\right), \text { for } \lambda<1, \\
f_{\gamma}\left(\theta \in\left[\theta_{+} ; \pi\right]\right)=\frac{C}{(1+\lambda \sin \theta)^{2}}\left(\frac{\tan \frac{\theta}{2}-\tan \frac{\theta_{+}}{2}}{\tan \frac{\theta}{2}-\tan \frac{\theta_{-}}{2}}\right)^{\frac{\lambda \pm \sqrt{\lambda^{2}-4}}{2 \sqrt{\lambda^{2}-1}}}, \text { for } \lambda>1 .
\end{gathered}
$$

These eigenmodes disappear at $\lambda>2 / \sqrt{3}$. This disappearance coincides with the steady state starting diverging at $\theta=$ $\theta_{+}+\epsilon$.
Finally, note that the eigenvalues $\gamma=\frac{1}{2}\left(-\lambda \pm \sqrt{\lambda^{2}-4}\right)$ for $\lambda<2$ correspond to solutions with an oscillatory be- 
havior superimposed with a decay in time, while for $\lambda>$ 2 they give steadily decaying in time solutions. This transition is identified in the paper in terms of the average qubit polarization, and has been identified in the detector's signal [42] as well. Here it appears as a property of the eigenvalues spectrum. Curiously, these eigenvalues are nonphysical for $\lambda>2 / \sqrt{3}$, which is before the transition is reached. At the same time, Eq. (6) does know about these eigenvalues for any $\lambda$, as we show in Appendix B2.
[1] K. Jacobs, Quantum Measurement Theory and its Applications (Cambridge University, Cambridge, England, 2014).

[2] H. Carmichael, An Open Systems Approach to Quantum Optics, Lecture Notes in Physics Monographs Vol. 18 (Springer-Verlag, Berlin, 1993), p. 179.

[3] C. Guerlin, J. Bernu, S. Deléglise, C. Sayrin, S. Gleyzes, S. Kuhr, M. Brune, Jean-michel Raimond, and S. Haroche, Progressive field-state collapse and quantum non-demolition photon counting, Nature (London) 448, 889 (2007).

[4] C. Sayrin, I. Dotsenko, X. Zhou, B. Peaudecerf, T. Rybarczyk, S. Gleyzes, P. Rouchon, M. Mirrahimi, H. Amini, M. Brune, J.-M. Raimond, and S. Haroche, Real-time quantum feedback prepares and stabilizes photon number states, Nature (London) 477, 73 (2011).

[5] K. W. Murch, S. J. Weber, C. Macklin, and I. Siddiqi, Observing single quantum trajectories of a superconducting quantum bit, Nature (London) 502, 211 (2013).

[6] K. W. Murch, S. J. Weber, K. M. Beck, E. Ginossar, and I. Siddiqi, Reduction of the radiative decay of atomic coherence in squeezed vacuum, Nature (London) 499, 62 (2013).

[7] S. J. Weber, A. Chantasri, J. Dressel, A. N. Jordan, K. W. Murch, and I. Siddiqi, Mapping the optimal route between two quantum states, Nature (London) 511, 570 (2014).

[8] R. Vijay, C. Macklin, D. H. Slichter, S. J. Weber, K. W. Murch, R. Naik, A. N. Korotkov, and I. Siddiqi, Stabilizing Rabi oscillations in a superconducting qubit using quantum feedback, Nature (London) 490, 77 (2012).

[9] M. S. Blok, C. Bonato, M. L. Markham, D. J. Twitchen, V. V. Dobrovitski, and R. Hanson, Manipulating a qubit through the backaction of sequential partial measurements and real-time feedback, Nat. Phys. 10, 189 (2014).

[10] G. de Lange, D. Ristè, M. J. Tiggelman, C. Eichler, L. Tornberg, G. Johansson, A. Wallraff, R. N. Schouten, and L. DiCarlo, Reversing Quantum Trajectories with Analog Feedback, Phys. Rev. Lett. 112, 080501 (2014).

[11] D. Ristè, M. Dukalski, C. A. Watson, G. de Lange, M. J. Tiggelman, Ya. M. Blanter, K. W. Lehnert, R. N. Schouten, and L. DiCarlo, Deterministic entanglement of superconducting qubits by parity measurement and feedback, Nature (London) 502, 350 (2013).

[12] N. Roch, M. E. Schwartz, F. Motzoi, C. Macklin, R. Vijay, A. W. Eddins, A. N. Korotkov, K. B. Whaley, M. Sarovar, and I. Siddiqi, Observation of Measurement-Induced Entanglement and Quantum Trajectories of Remote Superconducting Qubits, Phys. Rev. Lett. 112, 170501 (2014).

[13] J. P. Groen, D. Ristè, L. Tornberg, J. Cramer, P. C. de Groot, T. Picot, G. Johansson, and L. DiCarlo, Partial-Measurement Backaction and Nonclassical Weak Values in a Superconducting Circuit, Phys. Rev. Lett. 111, 090506 (2013).

[14] P. Campagne-Ibarcq, L. Bretheau, E. Flurin, A. Auffèves, F. Mallet, and B. Huard, Observing Interferences between Past and Future Quantum States in Resonance Fluorescence, Phys. Rev. Lett. 112, 180402 (2014).

[15] D. Tan, S. J. Weber, I. Siddiqi, K. Mølmer, and K. W. Murch, Prediction and Retrodiction for a Continuously Monitored Superconducting Qubit, Phys. Rev. Lett. 114, 090403 (2015).

[16] M. Naghiloo, D. Tan, P. M. Harrington, J. J. Alonso, E. Lutz, A. Romito, and K. W. Murch, Heat And Work Along Individual Trajectories of a Quantum Bit, Phys. Rev. Lett. 124, 110604 (2020).

[17] M. Naghiloo, J. J. Alonso, A. Romito, E. Lutz, and K. W. Murch, Information Gain and Loss for a Quantum Maxwell's Demon, Phys. Rev. Lett. 121, 030604 (2018).

[18] Y.-W. Cho, Y. Kim, Y.-H. Choi, Y.-S. Kim, S.-W. Han, S.-Y. Lee, S. Moon, and Y.-H. Kim, Emergence of the geometric phase from quantum measurement back-action, Nat. Phys. 15, 665 (2019).

[19] V. Gebhart, K. Snizhko, T. Wellens, A. Buchleitner, A. Romito, and Y. Gefen, Topological transition in measurement-induced geometric phases, Proc. Natl. Acad. Sci. USA 117, 5706 (2020).

[20] K. Snizhko, P. Kumar, N. Rao, and Y. Gefen, Weakmeasurement-induced asymmetric dephasing: A topological transition, arXiv:2006.13244 (2020).

[21] K. Snizhko, N. Rao, P. Kumar, and Y. Gefen, Weakmeasurement-induced phases and dephasing: broken symmetry of the geometric phase, arXiv:2006.14641 (2020).

[22] Y. Li, X. Chen, and M. P. A. Fisher, Quantum Zeno effect and the many-body entanglement transition, Phys. Rev. B 98, 205136 (2018).

[23] A. Chan, R. M. Nandkishore, M. Pretko, and G. Smith, Unitaryprojective entanglement dynamics, Phys. Rev. B 99, 224307 (2019).

[24] B. Skinner, J. Ruhman, and A. Nahum, Measurement-Induced Phase Transitions in the Dynamics of Entanglement, Phys. Rev. X 9, 031009 (2019).

[25] M. Szyniszewski, A. Romito, and H. Schomerus, Entanglement transition from variable-strength weak measurements, Phys. Rev. B 100, 064204 (2019).

[26] Z. K. Minev, S. O. Mundhada, S. Shankar, P. Reinhold, R. Gutiérrez-Jáuregui, R. J. Schoelkopf, M. Mirrahimi, H. J. Carmichael, and M. H. Devoret, To catch and reverse a quantum jump mid-flight, Nature (London) 570, 200 (2019).

[27] B. Misra and E. C. G. Sudarshan, The Zeno's paradox in quantum theory, J. Math. Phys. 18, 756 (1977).

[28] Asher Peres, Zeno paradox in quantum theory, Am. J. Phys. 48, 931 (1980).

[29] P. Facchi and S. Pascazio, Quantum Zeno Subspaces, Phys. Rev. Lett. 89, 080401 (2002).

[30] P. Facchi, A. G. Klein, S. Pascazio, and L. S. Schulman, Berry phase from a quantum Zeno effect, Phys. Lett. A 257, 232 (1999). 
[31] D. Burgarth, P. Facchi, V. Giovannetti, H. Nakazato, S. Pascazio, and K. Yuasa, Non-Abelian phases from quantum Zeno dynamics, Phys. Rev. A 88, 042107 (2013).

[32] S. Gherardini, S. Gupta, F. S. Cataliotti, A. Smerzi, F. Caruso, and S. Ruffo, Stochastic quantum Zeno by large deviation theory, New J. Phys. 18, 013048 (2016).

[33] T. J. Elliott and V. Vedral, Quantum quasi-Zeno dynamics: Transitions mediated by frequent projective measurements near the Zeno regime, Phys. Rev. A 94, 012118 (2016).

[34] M. Majeed and A. Z. Chaudhry, The quantum Zeno and antiZeno effects with non-selective projective measurements, Sci. Rep. 8, 14887 (2018).

[35] P. G. Kwiat, A. G. White, J. R. Mitchell, O. Nairz, G. Weihs, H. Weinfurter, and A. Zeilinger, High-Efficiency Quantum Interrogation Measurements via the Quantum Zeno Effect, Phys. Rev. Lett. 83, 4725 (1999).

[36] M. C. Fischer, B. Gutiérrez-Medina, and M. G. Raizen, Observation of the Quantum Zeno and Anti-Zeno Effects in an Unstable System, Phys. Rev. Lett. 87, 040402 (2001).

[37] J. Wolters, M. Strauß, R. S. Schoenfeld, and O. Benson, Quantum Zeno phenomenon on a single solid-state spin, Phys. Rev. A 88, 020101(R) (2013).

[38] A. Signoles, A. Facon, D. Grosso, I. Dotsenko, S. Haroche, J.-M. Raimond, M. Brune, and S. Gleyzes, Confined quantum Zeno dynamics of a watched atomic arrow, Nat. Phys. 10, 715 (2014).

[39] F. Schäfer, I. Herrera, S. Cherukattil, C. Lovecchio, F.S. Cataliotti, F. Caruso, and A. Smerzi, Experimental realization of quantum Zeno dynamics, Nat. Commun. 5, 3194 (2014).

[40] D. Layden, E. Martín-Martínez, and A. Kempf, Perfect Zeno-like effect through imperfect measurements at a finite frequency, Phys. Rev. A 91, 022106 (2015).
[41] M. Zhang, C. Wu, Y. Xie, W. Wu, and P. Chen, Quantum Zeno effect by incomplete measurements, Quantum Inf. Process. 18, 97 (2019).

[42] F. Li, J. Ren, and N. A. Sinitsyn, Quantum Zeno effect as a topological phase transition in full counting statistics and spin noise spectroscopy, Europhysics Lett. 105, 27001 (2014).

[43] C. Presilla, R. Onofrio, and U. Tambini, Measurement quantum mechanics and experiments on quantum Zeno effect, Ann. Phys. (NY) 248, 95 (1996)

[44] S. A. Gurvitz, L. Fedichkin, D. Mozyrsky, and G. P. Berman, Relaxation and the Zeno Effect in Qubit Measurements, Phys. Rev. Lett. 91, 066801 (2003).

[45] K. Koshino and A. Shimizu, Quantum Zeno effect by general measurements, Phys. Rep. 412, 191 (2005).

[46] A. Chantasri, J. Dressel, and A. N. Jordan, Action principle for continuous quantum measurement, Phys. Rev. A 88, 042110 (2013).

[47] P. Kumar, A. Romito, and K. Snizhko, The quantum Zeno effect with partial measurement and noisy dynamics, arXiv:2006.13970 (2020).

[48] R. Ruskov, A. Mizel, and A. N. Korotkov, Crossover of phase qubit dynamics in the presence of a negative-result weak measurement, Phys. Rev. B 75, 220501(R) (2007).

[49] J. Li, T. Wang, L. Luo, S. Vemuri, and Y. N. Joglekar, Unification of quantum Zeno-anti Zeno effects and parity-time symmetry breaking transitions, arXiv:2004.01364 (2020).

[50] Y.-H. Chen and T. A. Brun, Continuous quantum error detection and suppression with pairwise local interactions, arXiv:2004.07285 (2020).

[51] Y.-S. Kim, J.-C. Lee, O. Kwon, and Y.-H. Kim, Protecting entanglement from decoherence using weak measurement and quantum measurement reversal, Nat. Phys. 8, 117 (2012).

[52] In fact, it follows from Eq. (12) that $\partial_{\lambda} \bar{s}_{z}(t) \geqslant 0$ for any $t \geqslant 0$ when $\lambda>2$. 